\title{
Parabolic Diamond Scanning Probes for Single-Spin Magnetic Field Imaging
}

\author{
Natascha Hedrich®, Dominik Rohner, Marietta Batzer®, Patrick Maletinsky®, and \\ Brendan J. Shields $\oplus^{*}$ \\ Department of Physics, University of Basel, Klingelbergstrasse 82, Basel CH-4056, Switzerland
}

(Received 28 February 2020; revised 13 July 2020; accepted 21 September 2020; published 2 December 2020)

\begin{abstract}
Enhancing the measurement signal from solid-state quantum sensors such as the nitrogen-vacancy $(\mathrm{N}$ $V)$ center in diamond is a challenge for sensing and imaging of condensed-matter systems. Here we design and fabricate diamond scanning probes with a truncated parabolic profile that optimizes the photonic signal from single, near-surface, embedded $\mathrm{N}-V$ centers, forming a high-sensitivity probe for nanoscale magnetic field imaging. These structures operate over the full $\mathrm{N}-V$ photoluminescence spectrum with the emission being directed into a measured numerical aperture of 0.44 . Furthermore, they yield a median saturation count rate of $2.1 \mathrm{MHz}$, a fivefold improvement in measurement signal over the state of the art in scanning-probe-based $\mathrm{N}-V$ sensors. We additionally introduce a charge-state-registered pulsed-excitation method for measuring the detection efficiency of photons from the N- $V$ 's metrologically relevant negative charge state, finding an overall detection efficiency of $12 \%$. We demonstrate the excellent properties of these diamond scanning probes by imaging thin-film ferromagnetic stripes with a spatial resolution better than $50 \mathrm{~nm}$.
\end{abstract}

DOI: 10.1103/PhysRevApplied.14.064007

\section{INTRODUCTION}

Sensing and imaging of nanoscale sample volumes is increasingly relevant for a wide range of topics, from nanomagnetism to structural imaging in biology and condensed matter [1,2], and requires high sensitivity and high spatial resolution. Sensors based on solid-state defects such as the nitrogen-vacancy $(\mathrm{N}-V)$ center in diamond have emerged as ideal platforms for addressing such nanoscale phenomena [3], owing to the potential for sensor volumes approaching atomic dimensions. Optical initialization and readout of the $\mathrm{N}-V^{\prime}$ 's electronic spin [4,5] together with the ability to embed a single, near-surface $\mathrm{N}-V$ into all-diamond scanning-probe devices [6,7] have enabled the quantitative imaging of nanoscale systems such as skyrmions [8-10], domains in antiferromagnets $[11,12]$, electron transport in graphene [13,14], and magnetism in two-dimensional (2D) materials [15]. Despite these achievements, a wide range of even more challenging applications is currently beyond the sensitivity limits for practical scanning-probe-based studies [1,2], and points to the need for improved diamond scanning-probe technology.

\footnotetext{
*brendan.shields@unibas.ch

Published by the American Physical Society under the terms of the Creative Commons Attribution 4.0 International license. Further distribution of this work must maintain attribution to the author(s) and the published article's title, journal citation, and DOI.
}

Two aspects are key to the performance of $\mathrm{N}-V$-based nanoscale sensors and are addressed in this work. First, to minimize the $\mathrm{N}-V$-sample separation for optimal spatial resolution and to maximize the magnetic signal from nanoscale sample volumes, one requires a $\mathrm{N}-V$ center in close proximity to the diamond surface. Second, a high flux of detected photons from $\mathrm{N}-V$ photoluminescence (PL) is required to efficiently distinguish between the bright $m_{s}=|0\rangle$ and the dark $m_{s}=| \pm 1\rangle \mathrm{N}-V$ spin states for optimal magnetic sensitivity. The high refractive index of the diamond host $(n=2.4)$ presents a challenge for the second requirement, but also offers a natural route to engineer photonic structures that maximize PL detection.

Many approaches have been taken to optimize collection efficiency via photonic engineering of diamond. Microfabricated diamond solid immersion lenses [16,17] minimize oblique reflections at the diamond-air interface and have been successfully employed for entanglement of distant $\mathrm{N}-V \mathrm{~S}$ [18], with demonstrated PL detection rates of approximately $1 \mathrm{MHz}$. Approaches including bull's eye gratings [19], metalenses [20], and dielectric antennas [21] go a step further by redirecting in-plane emission towards a collection optic, with improved PL collection rates up to approximately $3.5 \mathrm{MHz}$ in the case of the bull's eye design [19]. However, the above approaches are targeted to quantum-information applications, where the $\mathrm{N}-V$ interacts only with nearby spins in the diamond lattice, and is embedded approximately $100 \mathrm{~nm}$ or more inside the diamond. This, along with the significant lateral extent of these structures, renders them 
impractical for scanning sensor applications, where the $\mathrm{N}-V$ must be brought close to a target external to the diamond.

For scanning-probe applications, the most practical device geometry is a single-sided waveguide, conventionally a cylindrical $[6,22]$ or tapered $[23,24]$ pillar. More recently, single-sided waveguides employing a parabolic pillar geometry and an oil-immersion imaging system have yielded the highest flux of photons of any structure [25], making them promising candidates for improving the sensitivity of scanning $\mathrm{N}-V$ magnetometry experiments. However, it remains an outstanding challenge to employ this highly efficient photonic structure in an oil-free scanningprobe configuration for nanoscale magnetometry, in part because of the large standoff distance between the diamond surface and the $\mathrm{N}-\mathrm{V}$ position at the focal point of the paraboloid.

In this work, we adapt the concept of the parabolic reflector to a scanning-probe geometry. We truncate the paraboloid apex to allow for small $\mathrm{N}-V$-sample spacings in scanning-probe imaging while maintaining the paraboloid's advantageous photonic properties. We further design the exit aperture of the device so as to direct the $\mathrm{N}-V$ emission into a small numerical aperture (NA), thereby minimizing reflections off the diamond-air interface at the output facet of the scanning probe. This yields a near-surface $\mathrm{N}-V$ in a high collection efficiency, broadband, waveguided device, thereby satisfying both of the requirements outlined above. We perform a detailed study of the $\mathrm{N}-V$ properties in these scanning probes to fully characterize the influence of the parabolic tip geometry on the photonic emission of the $\mathrm{N}-V$, including the $\mathrm{N}-V$ charge state and directionality of the probe emission. We develop a charge-state-registered pulsed-excitation measurement protocol to determine the photon-detection efficiency for photons emitted while the $\mathrm{N}-V$ resides in the metrologically relevant, negative charge state $\left(\mathrm{N}-V^{-}\right)$. Finally, we demonstrate the spatial resolution and sensitivity of these devices by imaging the magnetic field from a ferromagnetic Co-Fe-B stripline.

\section{CONCEPT AND SIMULATIONS}

Our scanning-probe design employs a truncated diamond paraboloid having an $\mathrm{N}-V$ at its focal point, which replaces the conventional cylindrical $[6,7]$ or tapered-pillar [24] geometry that acts to waveguide the N- $V$ PL. The intuition guiding our choice of a parabolic geometry [25] can be seen through a ray optics picture [Fig. 1(a)], where total internal reflection at the parabolic surface collimates the emission into a unidirectional output mode, resulting in improved waveguiding of the $\mathrm{N}-V$ emission. A useful feature of the design is the lateral expansion of the mode in the waveguide, which reduces diffraction of the mode as it exits the waveguide and results in a narrow (a)
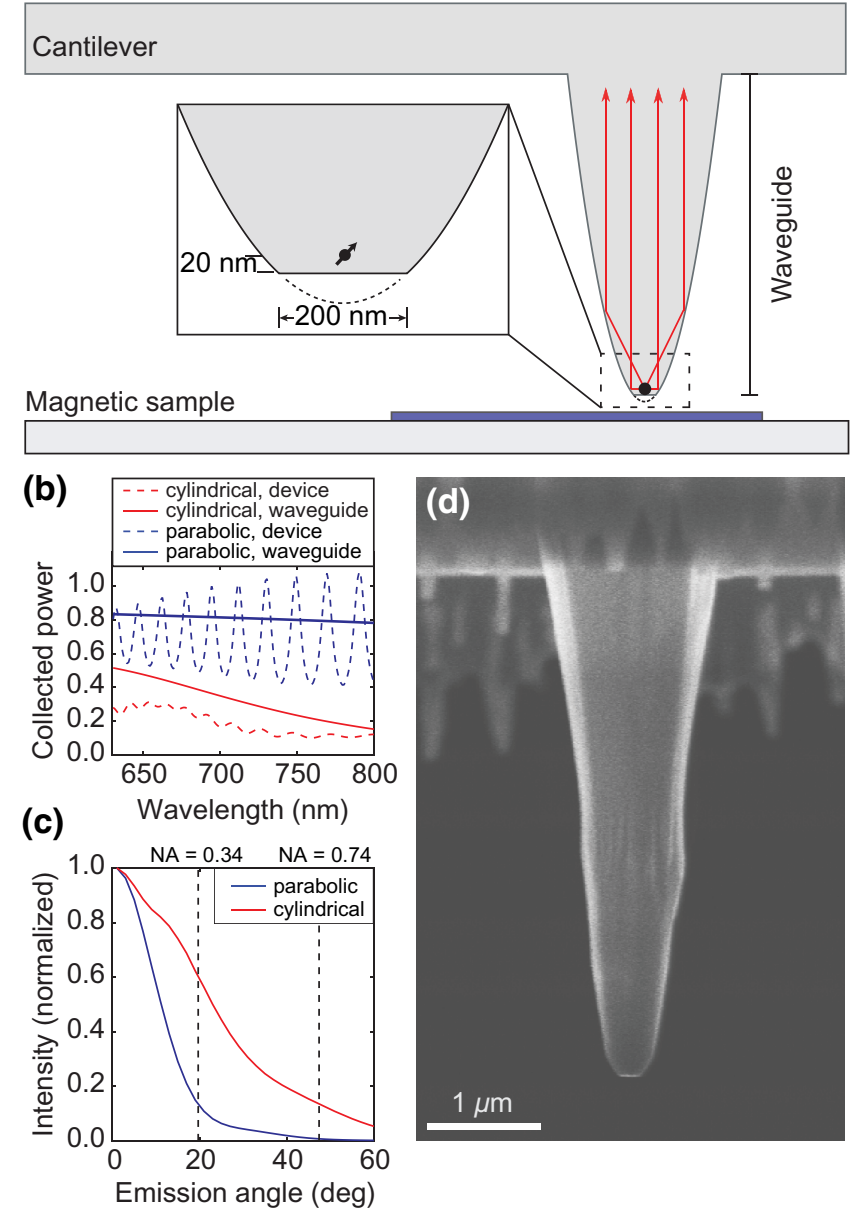

FIG. 1. Truncated parabolic scanning probe. (a) Diamond parabolic scanning-probe concept showing waveguiding of the emission of a $\mathrm{N}-V$ close to a magnetic surface. The inset shows the relevant length scales for the tip of the scanning probe. (b) Simulated device performance for cylindrical (red) and parabolic (blue) nanopillars showing the ratio of the power emitted by the waveguide into a NA $=0.8$, to the power emitted by a bulk dipole. This ratio is shown for the waveguide alone (solid lines), and the entire device (dashed lines) as a function of wavelength. (c) The normalized, integrated N- $V$ PL emitted from the device as a function of the polar exit angle, again for the cylindrical (red) and parabolic (blue) devices. (d) SEM image of completed pillar, consisting of parabolic tip and tapered waveguide.

spread in transverse wavevector, minimizing reflections at the diamond-air interface. The truncated design with a flat end facet minimizes the distance of the paraboloid's focal point, and hence the $\mathrm{N}-V$, from the sample.

We confirm this intuition by simulating the device with a finite-difference time-domain solver (Lumerical), taking a 200-nm-diameter cylindrical pillar waveguide as a basis of comparison [22]. The results are shown in Figs. 1(b) and 1(c) for a dipole transverse to the pillar axis and located on the axis at a depth of $20 \mathrm{~nm}$ from the facet. Additional 
simulation results, including those of a dipole oriented parallel to the waveguide, can be found in Ref. [26]. We first consider the power emitted into the waveguide without including outcoupling at the back side of the cantilever [cf. solid lines in Fig. 1(b)]. The parabolic device couples $81 \%$ of emitted power into the waveguide and has a flat response across the entire $\mathrm{N}-V$ emission spectrum $(630-800 \mathrm{~nm})$, whereas the cylindrical device is strongly chromatic and guides only $32 \%$ of the emitted power. The difference becomes even more pronounced when we consider the effect of outcoupling on the overall power emitted into the 0.8 NA cone of our objective [dashed lines in Fig. 1(b)]. In this case, the strongly confined mode in the cylindrical pillar has a large spread in transverse wavevector, resulting in the reflection and scattering into the cantilever of nearly half the waveguided power [26]. In contrast, the parabolic device mode is nearly normally incident at the back of the cantilever, resulting in the outcoupling of $83 \%$ of the waveguided power. The spread in transverse wavevector can be directly seen in Fig. 1(c), where we plot the far-field mode intensity as a function of polar angle for the two devices, averaged over azimuthal angle and over the $\mathrm{N}-V$ emission range. Overall, the parabolic device emits 4 times more power into the objective cone than the cylindrical device.

We realize the parabolic geometry by developing a scalable fabrication procedure based on electron-beam lithography and plasma-etching methods. In particular, we vary the mask erosion rate relative to the diamond etch rate, yielding precise control over the curvature of the final diamond device. We begin fabrication with a high-purity type-IIa diamond prepared with a layer of implanted N$V$ centers and prepatterned with an array of cantilevers etched to a depth of $2 \mu \mathrm{m}$. We then pattern $1-\mu \mathrm{m}$ diameter discs into an approximately 300 -nm-thick flowable oxide mask using electron-beam lithography. Etching of the pillars then consists of two plasma-etching stages in which we first develop the tapered diamond waveguide using an $\mathrm{O}_{2}$-based plasma, and secondly develop the parabolic tip by adding $\mathrm{CF}_{4}$ in increasing amounts. Raising the concentration of $\mathrm{CF}_{4}$ etches the mask more quickly, leading to a steeper angle of the diamond wall (the details of this process are given within the Supplemental Material [26-28]). A finished device is shown in Fig. 1(d).

\section{DEVICE CHARACTERIZATION}

We test the photonic performance of our devices in a homebuilt confocal microscope equipped with cw excitation lasers at 532 and $594 \mathrm{~nm}$, and a wavelength-tunable, supercontinuum picosecond pulsed laser [26]. We select 25 devices that contain a single $\mathrm{N}-V$ center for in-depth characterization.

\section{A. Charge-state corrected detection efficiency}

We first consider the PL intensity $I(P)$ of each device as a function of cw 532-nm excitation power $P$, which is the most common figure of merit for assessing photonic performance of single-N- $V$ devices. A PL intensity curve for a representative device is shown in Fig. 2(a), and clearly shows a saturation behavior as expected for a two-level system. We fit the saturation curve using

$$
I(P)=\frac{I_{\mathrm{PL}} P}{P+P_{\mathrm{sat}}}+I_{\mathrm{bg}} P,
$$

(a)

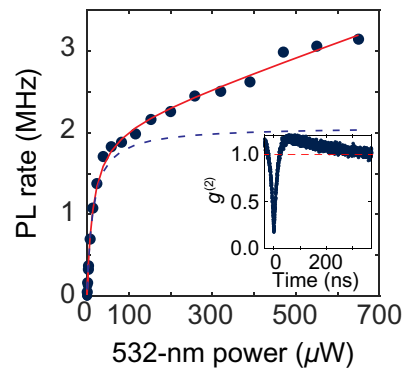

(c)

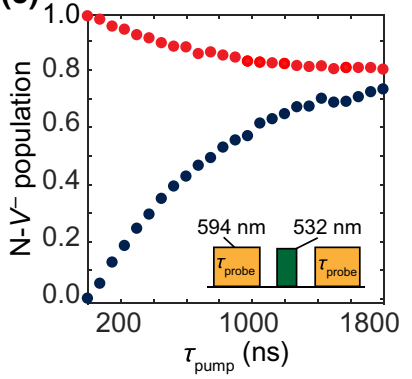

(b)

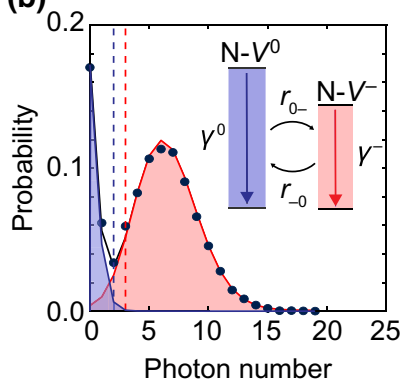

(d)

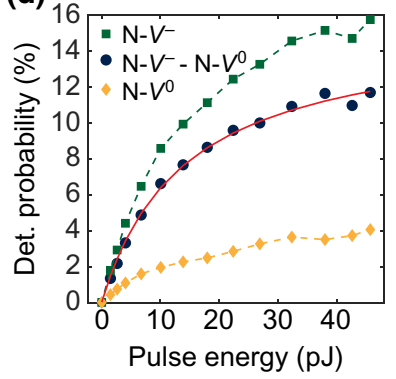

FIG. 2. Representative measurements to characterize the optical and $\mathrm{N}-V$-related properties of the fabricated devices. (a) Saturation curve taken with $\mathrm{cw} 532-\mathrm{nm}$ excitation resulting in $I_{\mathrm{PL}}=(2.1 \pm 0.2) \mathrm{MHz}$ and $P_{\text {sat }}=(14 \pm 3) \mu \mathrm{W}$. The blue dashed line shows the fitted fluorescence rate without the background. The inset shows a measured $g^{(2)}$ curve for this device, verifying that it is host to a single $\mathrm{N}-V$. (b) Photon-number distribution for a 594-nm probe pulse of duration $\tau_{\text {probe }}=250 \mu \mathrm{s}$. The $\mathrm{N}-V^{-}$and $\mathrm{N}-V^{0}$ thresholds are shown by red and blue lines, respectively, and the solid curves give fits to the distributions. The inset indicates the relevant $\mathrm{N}-V$ levels and transitions for describing the charge-state dynamics. (c) $\mathrm{N}-V^{-}$population measured by 594-nm excitation after starting in $\mathrm{N}-V^{0}$ (blue points) or $\mathrm{N}-V^{-}$(red points) for different 532-nm pulse lengths, converging to a steady-state $\mathrm{N}-V^{-}$population of $80 \pm 2 \%$. The inset shows the pulse sequence used for this measurement with yellow and green rectangles representing the 594- and 532-nm pulses, respectively. (d) The detection probability when starting and ending in $\mathrm{N}-V^{-}$(green squares) or $\mathrm{N}-V^{0}$ (yellow diamonds) as a function of the 532-nm excitation pulse energy. The two curves are subtracted (blue circles) to obtain the overall, backgroundcorrected detection probability. A saturation fit to the overall detection probability is shown in red. 
where $I_{\mathrm{PL}}$ is the saturated $\mathrm{PL}$ rate, $P_{\text {sat }}$ the saturation power, and $I_{\mathrm{bg}}$ the background intensity per unit excitation power. The contribution due to $\mathrm{N}-V \mathrm{PL}$ is indicated by the blue dashed line. Over the 25 structures investigated, the median PL intensity and saturation power are $I_{\mathrm{PL}}=2.1 \mathrm{MHz}$ and $P_{\text {sat }}=27 \mu \mathrm{W}$, with $1 \sigma$ confidence intervals of $[1.9,2.8] \mathrm{MHz}$ and $[20,48] \mu \mathrm{W}$, respectively [26]. These are, to our knowledge, the highest published $\mathrm{PL}$ intensities for N- $V$ centers in a scanning-probe geometry, and a factor of 5 improvement on previous realizations [24]. Additionally, the low saturation powers we find are generally advantageous, especially in cryogenic conditions where high laser powers can lead to heating.

While PL intensity is the most common figure of merit for assessing $\mathrm{N}-V$ photonic collection, it averages over PL fluctuations due to photoionization of the bright negative charge state $\left(\mathrm{N}-V^{-}\right)$to the dark neutral charge state $\left(\mathrm{N}-V^{0}\right)$ [29]. Most studies of N-V PL in high collection efficiency photonic devices overlook this significant and ubiquitous effect. To address this shortcoming, we measure the average $\mathrm{N}-V^{-}$population in our devices at saturation and show how to measure the true overall system detection efficiency by performing a high-fidelity initialization of the charge state, as we now describe.

The two $\mathrm{N}-V$ charge states can be optically distinguished via illumination with weak 594-nm excitation [29], since this excitation wavelength primarily leads to $\mathrm{N}-V^{-}$fluorescence and negligible optical excitation of $\mathrm{N}-V^{0}$. The number of photons counted during a 594-nm excitation pulse of duration $\tau_{\text {probe }}$, therefore, follows a bimodal distribution described by a two-state fluctuator model for N- $V$ chargestate dynamics [30] [Fig. 2(b)]. From this distribution, we extract the photoionization $\left(r_{-0}\right)$ and recombination $\left(r_{0_{-}}\right)$ rates, as well as the PL rates for $\mathrm{N}-V^{-}\left(\gamma^{-}\right)$and $\mathrm{N}-V^{0}\left(\gamma^{0}\right)$ under 594-nm illumination [26,31]. This allows us to set thresholds [dashed lines in Fig. 2(b)] for initializing the $\mathrm{N}-V$ into either charge state with high fidelity.

To measure the charge-state population at $I_{\text {sat }}$, we perform a pump-probe measurement using the sequence shown in the inset of Fig. 2(c). Using the thresholds, we assign the initial state to either $\mathrm{N}-V^{-}$or $\mathrm{N}-V^{0}$ based on the initial probe pulse, and record the photon-number histogram during the final probe pulse, depending on the initial state. This yields the final $\mathrm{N}-V^{-}$population as a function of initial charge state and pump duration, as shown in Fig. 2(c), where the 532-nm pump pulse is applied for a duration $\tau_{\text {pump }}$ and at power $P_{\text {sat }}$. With this, we determine the steady-state $\mathrm{N}-V^{-}$population under $532-\mathrm{nm}$ saturation conditions, which, for increasing $\tau_{\text {pump }}$, converges towards a median of $79 \%$ with a $1 \sigma$ confidence interval of $\mathrm{N}-V^{-} \in[71,82] \%$.

A similar procedure allows us to perform a measurement of the photon detection efficiency $\eta$ of our system, free of artifacts due to charge-state fluctuations. Typically, $\eta$ is measured by exciting the $\mathrm{N}-V$ with a short, intense pump pulse to transfer the population into the excited state, and by subsequently recording the fraction of excitations that result in a photon being recorded. However, the N- $V$ can also undergo ionization during the excitation pulse, resulting in no photon being detected. Additionally, in most high-collection efficiency photonic structures, establishing the background fluorescence level is complicated by the fact that the mode is highly localized, so it is not generally possible to measure a reference level for the same device in the absence of $\mathrm{N}-V$ fluorescence. We utilize the $\mathrm{N}-V$ charge state to eliminate these complications. We again perform a pump-probe experiment, for which the pump now consists of a single picosecond pulse provided by the supercontinuum source tuned to a spectral band between 590 and $620 \mathrm{~nm}$. We count the photons for $275 \mathrm{~ns}$ following the pump pulse, and record the likelihood to detect a photon as a function of pump power both for $\mathrm{N}-V^{-}$and $\mathrm{N}-V^{0}$, where we require the initial and final states to be either both $\mathrm{N}-V^{-}$, or both $\mathrm{N}-V^{0}$. As the pump pulse is outside the excitation band of $\mathrm{N}-V^{0}$, any photons detected from the $\mathrm{N}-V^{0}$ state [Fig. 2(d), yellow diamonds] are due to background fluorescence, allowing us to directly measure the background count level. This background is subtracted from the $\mathrm{N}-V^{-}$signal [Fig. 2(d), green squares] to obtain the final detection probability [Fig. 2(d), dark blue circles]. We measure a maximum detection probability of $\eta=12 \%$, which we report as the detection efficiency for this device.

This overall efficiency takes into account the parabolic reflector device efficiency $\eta_{\text {dev }}$ and the optical path efficiency $\eta_{\text {setup }}$, which we estimate to be $21 \%$ (including transmission through optical components, fiber coupling, and single photon counting module (SPCM) efficiency) [26]. Taking this value, we find a device collection efficiency of $\eta_{\text {dev }}=\eta / \eta_{\text {setup }}=57 \%$, which approaches the simulated value of $64 \%$ [26].

\section{B. Emission pattern}

In addition to the increased collection efficiency, we expect a spatial narrowing of the $\mathrm{N}-V$ emission, as compared to conventional, cylindrical pillars [Fig. 1(c)]. A reduced NA allows for improvements to the collection path, including antireflection coating of the diamond cantilever and collection lenses with lower NA, resulting in a larger working distance, and higher transmission, which would particularly facilitate cryogenic usage of scanning $\mathrm{N}-V$ magnetometers. We confirm this improved angularemission distribution via back focal plane (BFP) imaging of our scanning probes using an apparatus described in Ref. [32]. Figure 3(a) shows a BFP image of the PL from one of our devices, where the white dashed line indicates the NA $=0.8$ limit of the objective aperture. We fit the N$V$ emission with a two-dimensional Gaussian distribution plus a background [26], and from this obtain the contour at 

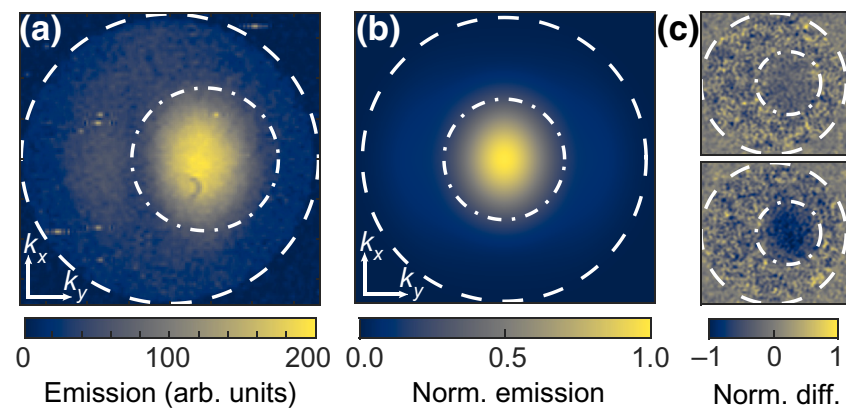

FIG. 3. Back focal plane (BFP) imaging of a representative pillar. (a) Waveguided emission into a NA $=0.37$ indicated by the dot-dashed line. The dashed line corresponds to the NA = 0.8 of our objective. (b) Simulations of the far-field emission coming from the backside of a parabolic device. The white dot-dashed line corresponds to a NA $=0.34$ [dashed line as in (a)]. (c) BFP images recorded while driving the $\mathrm{N}-V$ spin with microwave fields. Differential images off (top) and on (bottom) resonance, clearly show a dip in emission under $\mathrm{N}-V$ spin resonance and demonstrate that the corresponding emission pattern predominantly originates from $\mathrm{N}-V^{-}$emission.

which the emission intensity drops to $1 / e^{2}$ of its maximal value [white dash-dotted line in Fig. 3(a)].

From this fit, we extract a median best-fit emission $\mathrm{NA}_{\mathrm{BF}}=0.44$, and a $1 \sigma$ confidence interval of $[0.41,0.55]$. The simulated far-field emission pattern of a parabolic device is shown for comparison in Fig. 3(b), with the white dot-dashed line in representing the NA of 0.34 . The measured BFP image is notably off center, which we attribute to a slight tilt of the pillar from the sample normal, but is otherwise in good agreement with the simulated device. Furthermore, we utilize the BFP emission to show that our measurement signal indeed originates from the $\mathrm{N}-V$ by performing optically detected magnetic resonance (ODMR) while monitoring the BFP signal, as shown in Fig. 3(c). The two panels shows differential images taken by subtracting the BFP signal without microwave drive from the signal with microwave drive off resonance (top) and on resonance (bottom) with the $\mathrm{N}-V$ spin transition. Comparison of these two images clearly shows that the ODMR contrast is concentrated in the region of $\mathrm{NA}_{\mathrm{BF}}$.

\section{Applications for nanoscale magnetic field imaging}

The parabolic devices we present show great promise for scanning magnetic field imaging both in terms of sensitivity and spatial resolution. For the former, the sensitivity to magnetic fields derives from the ability to read out the $\mathrm{N}-V$ electronic spin optically. In Fig. 4(a), we show the pershot PL signal for a device undergoing Rabi nutations and subsequently optically driven by a $300-\mathrm{ns}, 532-\mathrm{nm}$ pulse at $4 P_{\text {sat }}$. The measured per-shot PL levels for the $|0\rangle$ and $| \pm 1\rangle$ states are $(0.479 \pm 0.003)$ and $(0.364 \pm 0.003)$, respectively, leading to a signal-to-noise ratio of $(0.125 \pm 0.004)$ (a)

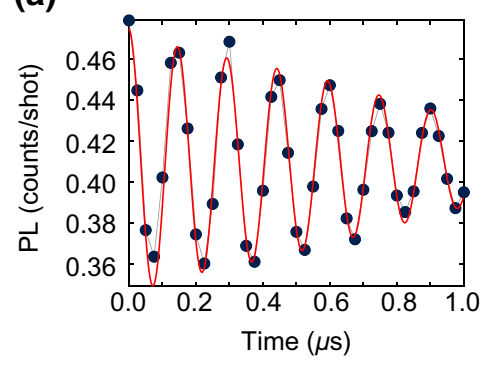

(b)

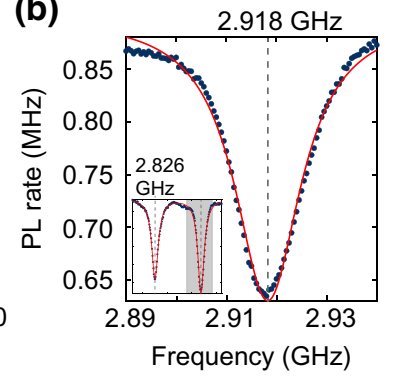

FIG. 4. Assessment of magnetic field sensitivity. (a) Microwave-driven Rabi nutations of the $\mathrm{N}-V$ spin for a representative device. The per-shot PL level for a 300-ns pulse of 532-nm illumination at $4 P_{\text {sat }}$ yields a signal-to-noise ratio of $0.125 \pm 0.004$ per shot. Each data point is averaged over 50000 repetitions, and the data is fit with a sum of exponentially decaying cosine functions (red). (b) cw ODMR taken at $P_{\text {sat }}$ for the same tip and fit with a Lorentzian (red). The inset shows the full ODMR spectrum with the position of the second resonance. The gray rectangle shows the location of the larger ODMR image.

[26,33]. Furthermore, to perform dc magnetometry, we measure a cw ODMR spectrum of the $\mathrm{N}-V$ spin transition [34], as shown in Fig. 4(b) for the same tip, driven at $P_{\text {sat }}$. From this ODMR, we extract the resulting de sensitivity of $(1.55 \pm 0.07) \mu \mathrm{T} / \sqrt{\mathrm{Hz}}$.

The second useful aspect for nanoscale imaging is the truncation of the parabola, which brings the N- $V$ close to the surface. This overcomes the primary drawback of other devices that aim to improve coupling to the $\mathrm{N}-V$ by placing the $\mathrm{N}-V$ at a focal point located deep in the diamond $[16,25]$. However, this proximity also means that $\mathrm{N}-V$ emission is affected by the presence of the nearby surface and its dielectric properties. To investigate this further, we simulate a parabolic device in contact with the surface of a dielectric substrate with varying index of refraction $n$ in Fig. 5(a), and observe that the power coupled into the waveguide is reduced for increasing $n$. We also confirm this behavior by measuring the ODMR [see Fig. 5(b)] of a fabricated device in air (red curve, out of contact) and on the surface of chromia, a dielectric with $n=2.6$ (blue curve, in contact). Here, we see the fluorescence drop from $0.8 \mathrm{MCts} / \mathrm{s}$ to $0.35 \mathrm{MCts} / \mathrm{s}$ - a drop of $56 \%$ - consistent with the expected drop in waveguided emission seen in Fig. 5(a).

We now demonstrate the general applicability of these devices by performing measurements of an out-of-plane magnetized ferromagnet using the same device mentioned in the previous paragraph. Specifically, we image a 1-nmthick, $0.73-\mu \mathrm{m}$-wide stripe of Co-Fe-B capped by a $5-\mathrm{nm}$ layer of Ta [Fig. 5(c)]. The measurement is performed in a homebuilt scanning-probe setup $[7,35]$ with a bias field of $28 \mathrm{G}$ applied along the $\mathrm{N}-V$ axis to split the relevant $\mathrm{N}-V$ spin states, $m_{s}=| \pm 1\rangle$, in energy. We then scan the N- $V$ in a direction perpendicular to the stripe, while recording a $\mathrm{cw}$ 


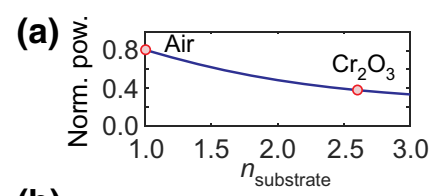

(b)

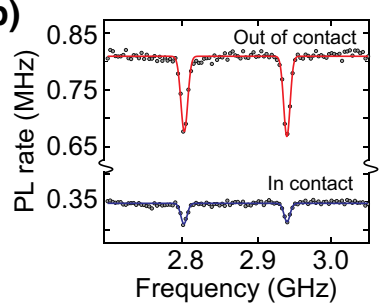

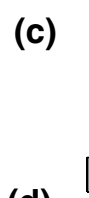

(d)

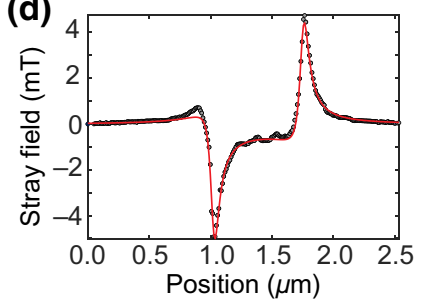

FIG. 5. Demonstration of scanning magnetometry with the truncated parabolic devices. (a) Simulated dipole power coupled to a parabolic waveguide, as a function of the index of refraction of a dielectric surface ( $\left.n_{\text {substrate }}\right)$, when in contact with the tip of the waveguide. The index of refraction for chromia $\left(\mathrm{Cr}_{2} \mathrm{O}_{3}\right.$, $n=2.6)$ and air $(n=1)$ are shown by circles. (b) ODMR taken for a fabricated parabolic device out of contact (red curve) and in contact (blue curve) with the surface of chromia. The data are shown as black points and the colored curves are Gaussian fits to the ODMR lines. (c) A schematic of the magnetic stripe together with the parabolic scanning probe. (d) The measured stray field along the $\mathrm{N}-V$ axis as a function of position. The fit (in red) is used to extract the $\mathrm{N}$ - $V$-to-sample distance $[(45 \pm 5) \mathrm{nm}]$ and the sample magnetization $[(1.0 \pm 0.2) \mathrm{mA}]$.

ODMR spectrum every $20 \mathrm{~nm}$ along the way. From this, we extract the stray magnetic field along the $\mathrm{N}-V$ axis using the Zeeman splitting of the $\mathrm{N}-V$ resonances, as shown in Fig. 5(d) (black circles).

The field of the ferromagnetic Co-Fe-B layer can be well described as arising from two oppositely flowing, onedimensional (1D) line currents running along the edge of the stripe [36]. These 1D features are excellent probes for the spatial resolution of our system, which appears as a broadening of the sharp peaks on either side of the stripe. As such, we fit the stray field with this line current model, and extract the sample magnetization $[(1.0 \pm 0.2) \mathrm{mA}]$ and the separation $[(45 \pm 5) \mathrm{nm}]$ between the $\mathrm{N}-V$ and the Co-Fe-B stripe. The latter determines the aforementioned broadening, and thus the spatial resolution of this type of scanning magnetometry [37,38], which is in this case $(45 \pm 5) \mathrm{nm}$. Thus, the truncated shape of our parabolic design helps to minimize the $\mathrm{N}-V$-sample separation, leading to a sub-50-nm spatial resolution, comparable with the state of the art at room temperature.

\section{CONCLUSION}

In conclusion, we fabricate parabolic diamond scanning probes containing single $\mathrm{N}-V$ centers and demonstrate their use for nanoscale magnetic field imaging. The parabolic design is ideal for sensing applications, as it yields a high

rate of detected photons from a near-surface $\mathrm{N}-V$. The devices can be further improved by incorporating an antireflection coating to the back surface of the cantilever to improve outcoupling, through the use of (111)-oriented diamond to achieve optimal mode overlap with the $\mathrm{N}-V$ optical transition dipoles [39-43], and via better lateral $\mathrm{N}-V$ placement by deterministic alignment to preselected $\mathrm{N}-V \mathrm{~s}$. Deterministic alignment would also allow for lower $\mathrm{N}$ implantation doses, a potential avenue to longer spin coherence times in these devices [26,44-46]. Our design is versatile and can be applied to many systems of interest, including scanning-probe sensing of magnetic and electric fields or temperature, as well as NMR sensing of molecules or materials attached to the diamond surface $[1,2]$.

\section{ACKNOWLEDGMENTS}

We thank M. Kasperczyk for fruitful discussions concerning the statistical analysis of our data. We further thank K. Garcia and R. Soucaille for fabrication of the Co-Fe-B sample investigated. We gratefully acknowledge financial support through the NCCR QSIT (Grant No. 185902), the Swiss Nanoscience Institute, the EU Quantum Flagship Project ASTERIQS (Grant No. 820394), and through the Swiss NSF Project Grants No. 188521 and No. 155845, and SNSF Spark Grant No. 190592.

N.H. conducted the majority of characterization and magnetometry measurements. B.J.S. conceived and oversaw the project, developed the fabrication and characterization methods, and performed simulations and measurements. B.J.S. and N.H. contributed equally to the fabrication of devices. D.R. and M.B. assisted with measurements. P.M. contributed to experimental methods. All authors prepared the manuscript.

[1] I. Lovchinsky, A. O. Sushkov, E. Urbach, N. P. de Leon, S. Choi, K. D. Greve, R. Evans, R. Gertner, E. Bersin, C. Müller, L. McGuinness, F. Jelezko, R. L. Walsworth, H. Park, and M. D. Lukin, Nuclear magnetic resonance detection and spectroscopy of single proteins using quantum logic, Science 351, 836 (2016).

[2] I. Lovchinsky, J. D. Sanchez-Yamagishi, E. K. Urbach, S. Choi, S. Fang, T. I. Andersen, K. Watanabe, T. Taniguchi, A. Bylinskii, E. Kaxiras, P. Kim, H. Park, and M. D. Lukin, Magnetic resonance spectroscopy of an atomically thin material using a single-spin qubit, Science 355, 503 (2017).

[3] F. Casola, T. van der Sar, and A. Yacoby, Probing condensed matter physics with magnetometry based on nitrogen-vacancy centres in diamond, Nature Reviews Materials 3, 1 (2018).

[4] A. Gruber, A. Dräbenstedt, C. Tietz, L. Fleury, J. Wrachtrup, and C. von Borczyskowski, Scanning confocal optical microscopy and magnetic resonance on single defect centers, Science 276, 2012 (1997). 
[5] M. W. Doherty, N. B. Manson, P. Delaney, F. Jelezko, J. Wrachtrup, and L. C. L. Hollenberg, The nitrogen-vacancy colour centre in diamond, Phys. Rep. 528, 1 (2013).

[6] P. Maletinsky, S. Hong, M. S. Grinolds, B. Hausmann, M. D. Lukin, R. L. Walsworth, M. Loncar, and A. Yacoby, A robust scanning diamond sensor for nanoscale imaging with single nitrogen-vacancy centres, Nat. Nanotechnol. 7, 320 (2012).

[7] P. Appel, E. Neu, M. Ganzhorn, A. Barfuss, M. Batzer, M. Gratz, A. Tschöpe, and P. Maletinsky, Fabrication of all diamond scanning probes for nanoscale magnetometry, Review of Scientific Instruments 87, 063703 (2016).

[8] A. Jenkins, M. Pelliccione, G. Yu, X. Ma, X. Li, K. L. Wang, and A. C. Bleszynski Jayich, Single-spin sensing of domain-wall structure and dynamics in a thinfilm skyrmion host, Physical Review Materials 3, 083801 (2019).

[9] Y. Dovzhenko, F. Casola, S. Schlotter, T. X. Zhou, F. Büttner, R. L. Walsworth, G. S. D. Beach, and A. Yacoby, Magnetostatic twists in room-temperature skyrmions explored by nitrogen-vacancy center spin texture reconstruction, Nat. Commun. 9, 1 (2018).

[10] I. Gross, W. Akhtar, A. Hrabec, J. Sampaio, L. J. Martínez, S. Chouaieb, B. J. Shields, P. Maletinsky, A. Thiaville, S. Rohart, and V. Jacques, Skyrmion morphology in ultrathin magnetic films, Phys. Rev. Mater. 2, 024406 (2018).

[11] I. Gross, W. Akhtar, V. Garcia, L. J. Martínez, S. Chouaieb, K. Garcia, C. Carrétéro, A. Barthélémy, P. Appel, P. Maletinsky, J. V. Kim, J. Y. Chauleau, N. Jaouen, M. Viret, M. Bibes, S. Fusil, and V. Jacques, Real-space imaging of non-collinear antiferromagnetic order with a single-spin magnetometer, Nature 549, 252 (2017).

[12] P. Appel, B. J. Shields, T. Kosub, N. Hedrich, R. Hübner, J. Faßbender, D. Makarov, and P. Maletinsky, Nanomagnetism of magnetoelectric granular thin-film antiferromagnets, Nano Lett. 19, 1682 (2019).

[13] M. J. H. Ku, T. X. Zhou, Q. Li, Y. J. Shin, J. K. Shi, C. Burch, L. E. Anderson, A. T. Pierce, Y. Xie, A. Hamo, U. Vool, H. Zhang, F. Casola, T. Taniguchi, K. Watanabe, M. M. Fogler, P. Kim, A. Yacoby, and R. L. Walsworth, Imaging viscous flow of the dirac fluid in graphene, Nature 583, 537 (2020).

[14] A. Jenkins, S. Baumann, H. Zhou, S. A. Meynell, D. Yang, K. Watanabe, T. Taniguchi, A. Lucas, A. F. Young, and A. C. Bleszynski Jayich, Imaging the breakdown of ohmic transport in graphene, arXiv:2002.05065.

[15] L. Thiel, Z. Wang, M. A. Tschudin, D. Rohner, I. Gutiérrez-Lezama, N. Ubrig, M. Gibertini, E. Giannini, A. F. Morpurgo, and P. Maletinsky, Probing magnetism in 2D materials at the nanoscale with single-spin microscopy, Science 364, 973 (2019).

[16] J. P. Hadden, J. P. Harrison, A. C. Stanley-Clarke, L. Marseglia, Y.-L. D. Ho, B. R. Patton, J. L. O’Brien, and J. G. Rarity, Strongly enhanced photon collection from diamond defect centers under microfabricated integrated solid immersion lenses, Appl. Phys. Lett. 97, 241901 (2010).

[17] M. Jamali, I. Gerhardt, M. Rezai, K. Frenner, H. Fedder, and J. Wrachtrup, Microscopic diamond solidimmersion-lenses fabricated around single defect centers by focused ion beam milling, Rev. Sci. Instrum. 85, 123703 (2014).
[18] B. Hensen, H. Bernien, A. E. Dréau, A. Reiserer, N. Kalb, M. S. Blok, J. Ruitenberg, R. F. L. Vermeulen, R. N. Schouten, C. Abellán, W. Amaya, V. Pruneri, M. W. Mitchell, M. Markham, D. J. Twitchen, D. Elkouss, S. Wehner, T. H. Taminiau, and R. Hanson, Loophole-free bell inequality violation using electron spins separated by 1.3 kilometres, Nature 526, 682 (2015).

[19] L. Li, E. H. Chen, J. Zheng, S. L. Mouradian, F. Dolde, T. Schröder, S. Karaveli, M. L. Markham, D. J. Twitchen, and D. Englund, Efficient photon collection from a nitrogen vacancy center in a circular bullseye grating, Nano Lett. 15, 1493 (2015).

[20] T.-Y. Huang, R. R. Grote, S. A. Mann, D. A. Hopper, A. L. Exarhos, G. G. Lopez, G. R. Kaighn, E. C. Garnett, and L. C. Bassett, A monolithic immersion metalens for imaging solid-state quantum emitters, Nat. Commun. 10, 1 (2019).

[21] D. Riedel, D. Rohner, M. Ganzhorn, T. Kaldewey, P. Appel, E. Neu, R. J. Warburton, and P. Maletinsky, Low-Loss Broadband Antenna for Efficient Photon Collection from a Coherent Spin in Diamond, Phys. Rev. Appl. 2, 064011 (2014).

[22] B. J. M. Hausmann, M. Khan, Y. Zhang, T. M. Babinec, K. Martinick, M. McCutcheon, P. R. Hemmer, and M. Lončar, Fabrication of diamond nanowires for quantum information processing applications, Diam. Relat. Mater. 19, 621 (2010).

[23] S. A. Momenzadeh, R. J. Stöhr, F. F. de Oliveira, A. Brunner, A. Denisenko, S. Yang, F. Reinhard, and J. Wrachtrup, Nanoengineered diamond waveguide as a robust bright platform for nanomagnetometry using shallow nitrogen vacancy centers, Nano Lett. 15, 165 (2015).

[24] T. X. Zhou, R. J. Stöhr, and A. Yacoby, Scanning diamond NV center probes compatible with conventional AFM technology, Appl. Phys. Lett. 111, 163106 (2017).

[25] N. H. Wan, B. J. Shields, D. Kim, S. Mouradian, B. Lienhard, M. Walsh, H. Bakhru, T. Schröder, and D. Englund, Efficient extraction of light from a nitrogen-vacancy center in a diamond parabolic reflector, Nano Lett. 18, 2787 (2018).

[26] See Supplemental Material at http://link.aps.org/supple mental/10.1103/PhysRevApplied.14.064007 for simulation details, fabrication procedure, description of setup and estimation of losses, measurement details, PL lifetime measurements, measurement statistics, back focal plane fitting, sensitivity and $T_{2}$ measurements.

[27] Y. Chu, N. P. de Leon, B. J. Shields, B. Hausmann, R. Evans, E. Togan, M. J. Burek, M. Markham, A. Stacey, A. S. Zibrov, A. Yacoby, D. J. Twitchen, M. Loncar, H. Park, P. Maletinsky, and M. D. Lukin, Coherent optical transitions in implanted nitrogen vacancy centers, Nano Lett. 14, 1982 (2014).

[28] T. Yamada, H. Yoshikawa, H. Uetsuka, S. Kumaragurubaran, N. Tokuda, and S.-I. Shikata, Cycle of two-step etching process using ICP for diamond MEMS applications, Diam. Relat. Mater. 16, 996 (2007).

[29] N. Aslam, G. Waldherr, P. Neumann, F. Jelezko, and J. Wrachtrup, Photo-induced ionization dynamics of the nitrogen vacancy defect in diamond investigated by singleshot charge state detection, New J. Phys. 15, 013064 (2013). 
[30] B. J. Shields, Q. P. Unterreithmeier, N. P. de Leon, H. Park, and M. D. Lukin, Efficient Readout of a Single Spin State in Diamond via Spin-To-Charge Conversion, Phys. Rev. Lett. 114, 136402 (2015).

[31] D. Bluvstein, Z. Zhang, and A. C. Bleszynski Jayich, Identifying and Mitigating Charge Instabilities in Shallow Diamond Nitrogen-Vacancy Centers, Phys. Rev. Lett. 122, 076101 (2019).

[32] D. Rohner, Master's thesis, Universität Basel, 2013.

[33] D. A. Hopper, H. J. Shulevitz, and L. C. Bassett, Spin readout techniques of the nitrogen-vacancy center in diamond, Micromachines 9, 437 (2018).

[34] J. F. Barry, J. M. Schloss, E. Bauch, M. J. Turner, C. A. Hart, L. M. Pham, and R. L. Walsworth, Sensitivity optimization for NV-diamond magnetometry, Rev. Mod. Phys. 92, 015004 (2020).

[35] P. Appel, M. Ganzhorn, E. Neu, and P. Maletinsky, Nanoscale microwave imaging with a single electron spin in diamond, New J. Phys. 17, 112001 (2015).

[36] J.-P. Tetienne, T. Hingant, L. J. Martínez, S. Rohart, A. Thiaville, L. H. Diez, K. Garcia, J.-P. Adam, J.-V. Kim, J.-F. Roch, I. M. Miron, G. Gaudin, L. Vila, B. Ocker, D. Ravelosona, and V. Jacques, The nature of domain walls in ultrathin ferromagnets revealed by scanning nanomagnetometry, Nat. Commun. 6, 6733 (2015).

[37] R. Schirhagl, K. Chang, M. Loretz, and C. L. Degen, Nitrogen-vacancy centers in diamond: Nanoscale sensors for physics and biology, Annu. Rev. Phys. Chem. 65, 83 (2014).

[38] S. K. Sekatskii and V. S. Letokhov, Nanometer-resolution scanning optical microscope with resonance excitation of the fluorescence of the samples from a single-atom excited center, J. Exp. Theor. Phys. Lett. 63, 319 (1996).

[39] D. Rohner, J. Happacher, P. Reiser, M. A. Tschudin, A. Tallaire, J. Achard, B. J. Shields, and P. Maletinsky, (111)-Oriented, single crystal diamond tips for nanoscale scanning probe imaging of out-of-plane magnetic fields, Appl. Phys. Lett. 115, 192401 (2019).

[40] W. Lukosz and R. E. Kunz, Light emission by magnetic and electric dipoles close to a plane dielectric interface. II. Radiation patterns of perpendicular oriented dipoles, J. Opt. Soc. Am. 67, 1615 (1977).

[41] R. J. Epstein, F. M. Mendoza, Y. K. Kato, and D. D. Awschalom, Anisotropic interactions of a single spin and dark-spin spectroscopy in diamond, Nat. Phys. 1, 94 (2005).

[42] T. P. M. Alegre, C. Santori, G. Medeiros-Ribeiro, and R. G. Beausoleil, Polarization-selective excitation of nitrogen vacancy centers in diamond, Phys. Rev. B 76, 165205 (2007).

[43] L. Robledo, H. Bernien, T. van der Sar, and R. Hanson, Spin dynamics in the optical cycle of single nitrogen-vacancy centres in diamond, New J. Phys. 13, 025013 (2011).

[44] E. L. Hahn, Free nuclear induction, Phys. Today 6, 4 (1953).

[45] P. L. Stanwix, L. M. Pham, J. R. Maze, D. Le Sage, T. K. Yeung, P. Cappellaro, P. R. Hemmer, A. Yacoby, M. D. Lukin, and R. L. Walsworth, Coherence of nitrogenvacancy electronic spin ensembles in diamond, Phys. Rev. B 82, 201201 (2010).

[46] R. Hanson, V. V. Dobrovitski, A. E. Feiguin, O. Gywat, and D. D. Awschalom, Coherent dynamics of a single spin interacting with an adjustable spin bath, Science 320, 352 (2008). 